\title{
Performance Evaluation of Phase Separation Process Using High-concentration AMP Promoted by MAPA for $\mathrm{CO}_{2}$ Capture
}

\author{
Akihisa MAtsui ${ }^{1}$, Naoya OGIYAMA ${ }^{1}$, Takumi ENDO ${ }^{2}$, Jun ARAKAWA ${ }^{2}$, and Takao NAKAGAKI ${ }^{1}$ \\ ${ }^{1}$ Depertment of Modern Mechanical Engineering, Waseda University, 3-4-1 Okubo, Shinjuku-ku, Tokyo 160-8555, Japan \\ ${ }^{2}$ Business Development Dept. Resources, Energy \& Environment Business Area, IHI Corporation, 3-1-1 Toyosu, Koto-ku, Tokyo 135- \\ 8710 , Japan
}

\begin{abstract}
Reduction of the energy penalty and cost of $\mathrm{CO}_{2}$ capture from concentrated gas streams using amine-based solutions can be achieved by minimizing the energy penalty in the solvent regeneration process. High concentration 2-Amino-2-methyl-1-propanol (AMP) solution precipitates as a carbonate when enough $\mathrm{CO}_{2}$ has been absorbed. By sending the separated carbonate to the stripper, the sensible heat of regeneration can be reduced. However, previous testing using 50 weight percent AMP solution mixed with Piperazine (PZ) with solid-liquid separation showed that the $\mathrm{CO}_{2}$ recovery rate was limited to $65 \%$ due to the lack of $\mathrm{PZ}$ regeneration. To improve the $\mathrm{CO}_{2}$ recovery rate, a novel solution and injection process were developed. N-Methyl-1,3-diaminopropane (MAPA) was selected as an alternative promoter based on reaction rate testing. Various tests were employed to characterize the behaviour of the AMP/MAPA solution under $\mathrm{CO}_{2}$ capture and recovery conditions. The injection point was relocated to avoid the inhibition of $\mathrm{CO}_{2}$ absorption observed when $\mathrm{CO}_{2}$ semi-lean liquid was sent to the upper portion of the absorber. The $\mathrm{CO}_{2}$ recovery rate and the precipitation quantity were simulated using a model built in Aspen Plus ${ }^{\circledR}$. The novel solution and injection set-up were evaluated experimentally by a bench-scale apparatus.
\end{abstract}

\section{Introduction}

Carbon Capture and Storage (CCS) is widely considered as a necessary technology to meet the Paris Agreement's "well below $2^{\circ} \mathrm{C}$ above pre-industrial levels and pursuing efforts to limit the temperature increase to $1.5^{\circ} \mathrm{C}$ " (UNFCCC, 2015; IPCC, 2018). For capture of $\mathrm{CO}_{2}$ from large scale emission point sources (e.g., thermal power stations), chemical absorption is the most widely studied and mature technology. While technically proven, the chemical absorption method has the drawback of a large energy penalty owing to the regeneration of the solvents used to capture $\mathrm{CO}_{2}$. To maintain financial viability while reducing $\mathrm{CO}_{2}$ emissions, further process improvement and innovation are warranted. Regeneration heat is roughly divided into three sources: sensible heat, heat of vaporization, and heat of dissociation. The sensible heat is expected to be reduced via a phase separation of $\mathrm{CO}_{2}-$ rich and $\mathrm{CO}_{2}$-lean amine. By sending only the $\mathrm{CO}_{2}$-rich phase to stripper, the quantity of solution circulating in stripper decrease, thereby reducing the sensible heat load.

Phase separation processes commonly researched in the CCS chemical absorption field are chilled ammonia method, liquid-liquid separation method, and solid-liquid separation method. This study utilizes the solid-liquid separation method due to its higher safety and more rapid kinetics. In the solid-liquid separation process, a high concentration amine solution is used and a precipitated solid of protonated 2-Amino-2-methyl-1-propanol (AMP) carbonate (henceforth: carbonate) is formed. Only this solid carbonate is sent to stripper (Nakagaki et al., 2013).

High concentration AMP solution of $\geq 40 \mathrm{wt} \%$ results in precipitation of solid carbonate upon $\mathrm{CO}_{2}$ absorption. The $\mathrm{CO}_{2}$ absorption rate of AMP is slow due to steric hindrance. However, the absorption rate of AMP is accelerated by adding a small quantity of amine with a high reaction rate with $\mathrm{CO}_{2}$ (e.g., Piperazine: PZ) as a promoter (Ying et al., 2017). To confirm whether the such results remain in solid-liquid type processes, $\mathrm{CO}_{2}$ capture and recovery tests were performed using AMP 50wt $\%$ promoted with $\mathrm{PZ} 5 \mathrm{wt} \%$.

Figure 1 shows the operating conditions over the process steps of the $\mathrm{CO}_{2}$ capture and recovery test (Nakagaki et al, 2015). The arrow corresponds to each unit operation ordered by ordinal numbers which indicate condition of the $\mathrm{CO}_{2}$ loading and temperature of the circulating solution. A solvent rich in $\mathrm{CO}_{2}$ is cooled at the bottom of the absorber column and precipitates as carbonates. A slurry of solid carbonates mixed with a small amount of liquid amine is separated by a filter-type centrifuge, the solids are dissolved by a mantle heater, and

* Corresponding author: pineforest@toki.waseda.jp 
pumped to the stripper. The separated liquid phase $\left(\mathrm{CO}_{2}\right.$ semi-lean liquid) is mixed with lean liquid regenerated in the stripper and returned to the upper portion of the absorber column. In our previous study, the operating conditions and equipment setup to prevent blockage by precipitates were designed (Teranishi et al., 2016) and using this setup with AMP 50wt\% promoted with $\mathrm{PZ}$ $5 \mathrm{wt} \%$, the sensible heat decreased by $15-27 \%$ compared to a liquid phase process using AMP 30wt\% (Ogiyama et al., 2017). However, the $\mathrm{CO}_{2}$ recovery rate was limited to $65 \%$ due to the lack of regeneration of $\mathrm{PZ}$ in the stripper.

This study aimed to improve the $\mathrm{CO}_{2}$ recovery rate by searching for effective promoter of absorbent and modifying process configuration. The candidates of effective promoter were selected from amines reported in the literature which were expected to have high regeneration performance under conditions like this phase-separation process. These amines were blended with AMP and the basic characteristics of new blended amines were evaluated. As for process modifications, the injection of separated semi-lean liquid, which is usually injected into the top of the absorber, was relocated to the middle of absorber column. The rationale for this alteration was to maintain the $\mathrm{CO}_{2}$ absorption driving force in the upper part of the column. The alteration to the regeneration heat and $\mathrm{CO}_{2}$ recovery rate using new blended amine solutions and the new equipment set-up were empirically evaluated.

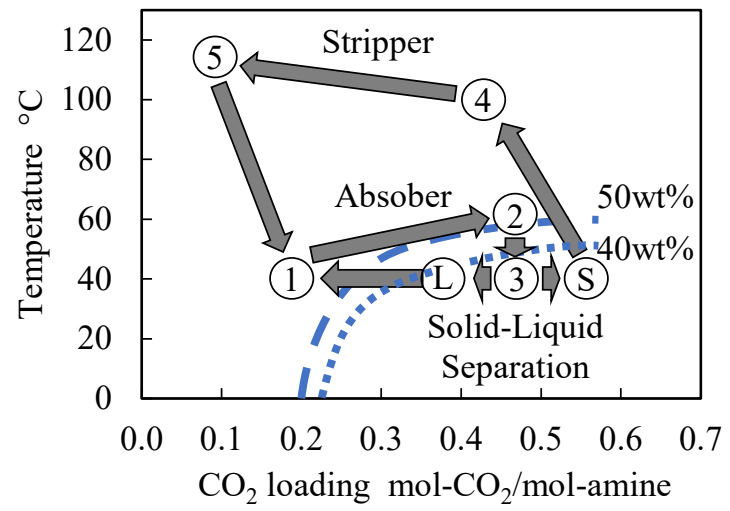

Figure 1. Process operation line of $\mathrm{CO}_{2}$ recovery test using AMP $50 \mathrm{wt} \%+\mathrm{PZ} 5 \mathrm{wt} \%$. (1) is the top of absorber column, (2) is the bottom of absorber column, (3) is the centrifuge, (4) is the top of stripper column and (5) is the bottom of stripper column, L is semi-lean liquid, and $\mathrm{S}$ is carbonate. Two lines are boundary of precipitation in AMP 50wt $\%$ and AMP $40 \mathrm{wt} \%$.

\section{Promoter Selection and Solution Property Evaluation}

\subsection{Selection of promoters based on reaction rate testing}

Amines possible to react as promoter were gleaned from the literature (Bernhardsen et al, 2017a, 2017b) and blended with AMP to test their performance impact.
Specifically, 1,6-Diaminohexane (HMDA), 1,4Diaminobutane (DAB), N-(2-Aminoethyl) piperazine (AEP), Benzylamine (BZA), and N-Methyl-1,3diaminopropane (MAPA) were evaluated. Figure 2 provides a schematic of the reaction rate test apparatus (Inoue et al., 2013). A mixture of gaseous $\mathrm{CO}_{2}$ and $\mathrm{N}_{2}$ is supplied into the heated reactor, and the reaction rate is calculated by measuring the $\mathrm{CO}_{2}$ concentration of the outlet gas, yielding the $\mathrm{CO}_{2}$ absorbed per unit area. Figure 3 shows results of the testing. Although the addition of HMDA, DAB, AEP, and BZA did not accelerate the reaction rate, MAPA significantly improved the reaction rate. It has been reported that MAPA forms an eightmember ring which is easily regenerated and improves the absorption rate (Zhang et al., 2018). Based on the above results, MAPA was selected as a promoter.

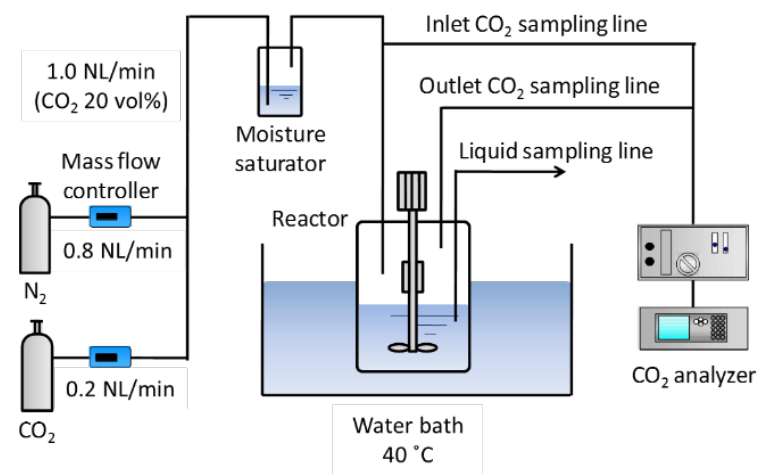

Figure 2. Static gas-liquid contactor measuring reaction rate

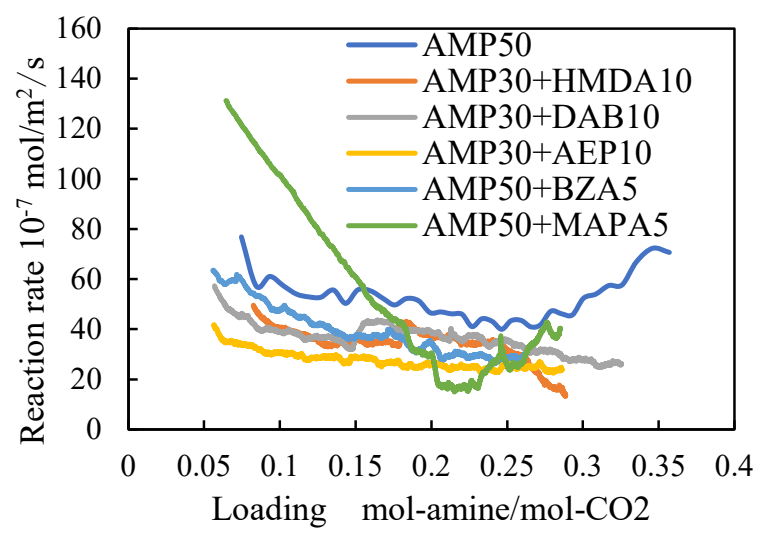

Figure 3. Results of reaction rate of various AMP-based solutions blended with promoters

\subsection{Vapor-liquid equilibrium test}

The vapor-liquid equilibrium characteristics of AMP $50 \% /$ MAPA $5 \%$ solution were tested in the temperature range of the absorber and stripper columns. Figure 4 shows the results of test and the results of Dash et al. (2011) using AMP $43 \mathrm{wt} \%$ at $55^{\circ} \mathrm{C}$. The $\mathrm{CO}_{2}$ loading of solution was measured by the total organic carbon analyzer. From the fact that the results of Dash et al. (2011) fall between the $50^{\circ} \mathrm{C}$ and $60^{\circ} \mathrm{C}$ VLE plots of this test, it was concluded that the VLE behaviour of AMP was not significantly influenced by the addition of MAPA. 


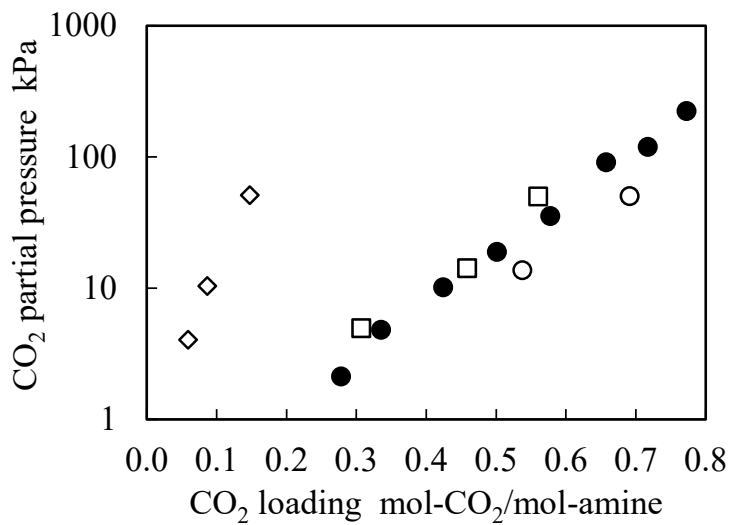

Figure 4. VLE curves of AMP 50wt $\%+$ MAPA $5 \mathrm{wt} \%$ solution. $\circ, 50^{\circ} \mathrm{C} ; \square, 60^{\circ} \mathrm{C} ; \diamond, 120^{\circ} \mathrm{C}$; and $\bullet$, Dash et al. $(2011)$ $\mathrm{AMP}=43 \mathrm{wt} \%$ at $55^{\circ} \mathrm{C}$

\subsection{Measurement of precipitation and dissolution temperature}

Precipitation and dissolution temperature of carbonate were measured by heating and cooling solution in a glass container to determine the precipitation boundary of AMP/MAPA solution selected as described in section 2.1. $\mathrm{CO}_{2}$ loading of solution was $0.15-0.45\left(\mathrm{~mol}-\mathrm{CO}_{2} / \mathrm{mol}-\right.$ amine). Figure 5 shows the measurement result and the precipitation boundary against the temperature-dependent loading. Precipitation can be avoided by maintaining a temperature $>60^{\circ} \mathrm{C}$ under a $\mathrm{CO}_{2}$ partial pressure of $15 \mathrm{kPa}$.

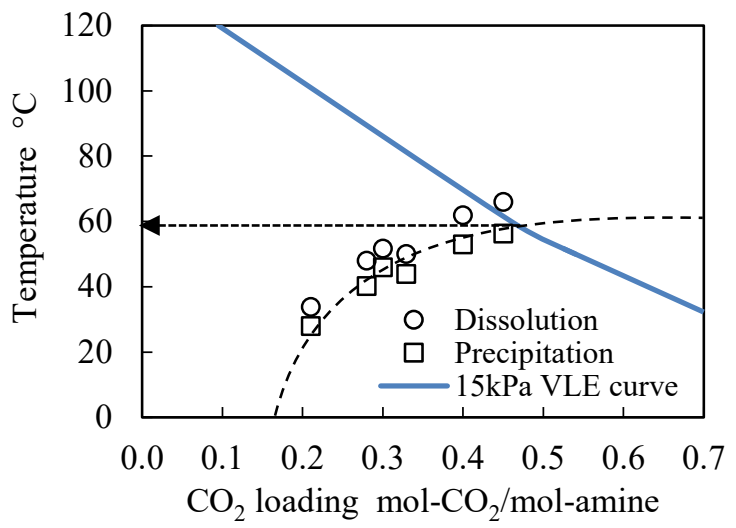

Figure 5. Boundary of precipitation against temperature and $\mathrm{CO}_{2}$ loading. Blue line is VLE curve of $\mathrm{CO}_{2}$ partial pressure $15 \mathrm{kPa}$.

\subsection{Cooling precipitation test}

The cooling temperature required to induce carbonate precipitation was obtained by batch testing. The theoretical formula of Kubota (Kobari, 2014) is shown in Eq. (1), where $\Delta T_{\text {ind }}$ is the degree of supersaturation, $(N / M)_{m}$ is the assumed number density of primary nuclei for detection sensitivity $k_{\mathrm{b} 1}$ is the primary nucleation constant, $n$ is nucleation order, and $\Delta T$ is the degree of supercooling.

$$
\log \left(\Delta T_{\text {ind }}\right)=\log \left[(N / M)_{m} / k_{\mathrm{b} 1}\right]-n \log (\Delta T)
$$

Logarithmic plots of $\Delta T$ and lag time to precipitation are linear as shown in Eq. (1), and $k_{\mathrm{b} 1}$ can be determined by the slope and intercept (isothermal method). After quenching $5 \mathrm{~mL}$ AMP $50 \mathrm{wt} \%$ solution, the temperature was held constant and the lag time to carbonate precipitation was measured. The primary nucleation constant was calculated from the measured time, the supersaturation degree, and the cooling temperature in the $\mathrm{CO}_{2}$ capture and recovery test. The assumed number density of primary nuclei for detection sensitivity was set to $(N / M)_{m}=500$. Figure 6 shows the results of testing, which indicates the waiting time for precipitation against the degree of supersaturation become shorter as the $\mathrm{CO}_{2}$ loading increase. Calculating $k_{\mathrm{b} 1}$ and $n$ in Eq. (1) by the plots and substituting waiting time of solution in cooling container into Eq. (1) gives the required degree of supersaturation for precipitation. The $\mathrm{CO}_{2}$ loading in the bottom of absorber was assumed to be 0.45 , and the waiting time of solution in cooling container was 2010 seconds, so the degree of supersaturation was determined to be $6.4^{\circ} \mathrm{C}\left(T=53.6^{\circ} \mathrm{C}\right)$.

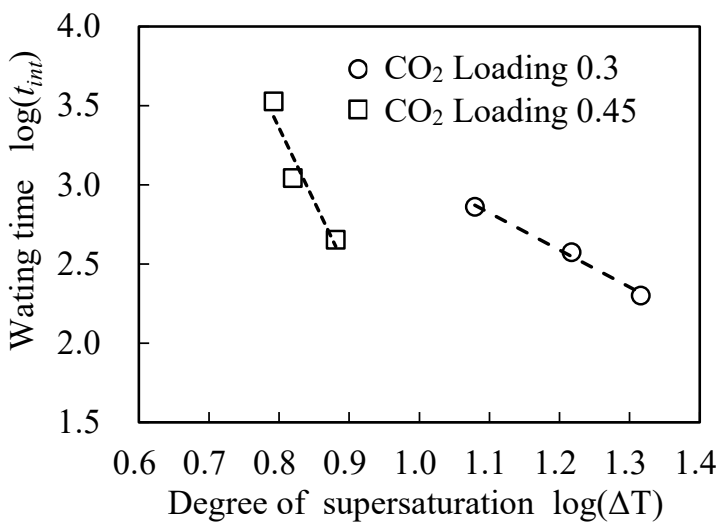

Figure 6. Waiting time for precipitation measured by isothermal method

\subsection{Carbonate analysis}

If MAPA is contained in the precipitates, then regeneration heat must be examined considering this MAPA-carbonate structure. Carbonate was analysed by Raman spectroscopy and gas chromatography and mass spectrometry (GC-MS) to confirm whether MAPA was contained in the precipitate. Figure. 7 shows the Raman spectra of the precipitates from AMP $50 \mathrm{wt} \%$, AMP 50 wt $\%+$ MAPA $5 \mathrm{wt} \%$, pure AMP, and pure MAPA. Although peaks commonly observed for hydrocarbons and AMP were detected in the spectrum for AMP+MAPA, no peaks specific to MAPA were not detected (see Figure 7).

Figure 8 shows the analysis results of AMP 50 wt $\%+$ MAPA $5 \mathrm{wt} \%$, AMP $100 \%$, and MAPA $100 \%$ by GC-MS. Spectra of pure AMP and AMP $50 \mathrm{wt} \%+$ MAPA $5 \mathrm{wt} \%$ were observed at an elapsed period of 6.6 minutes, the peak of pure MAPA was observed at a period of 7.4 
minutes. According to the above result, the carbonate precipitated in AMP/MAPA solution did not contain any salts derived from MAPA.

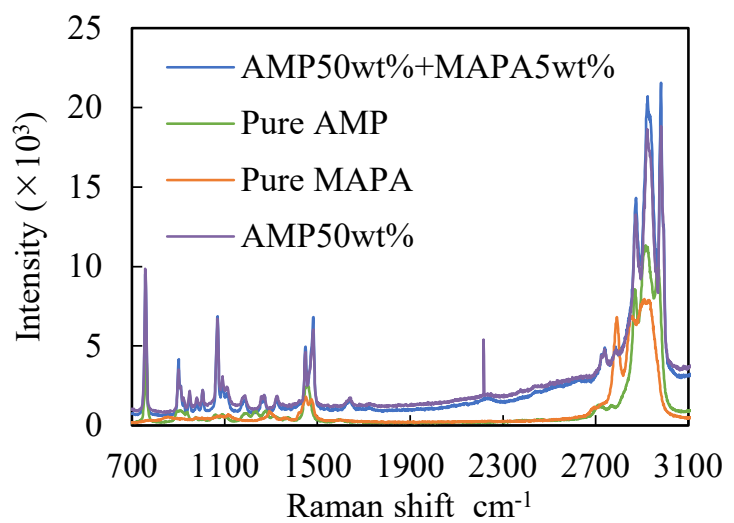

Figure 7. Raman spectra of the precipitates from the AMP 50 wt $\%+$ MAPA $5 \mathrm{wt} \%$. For comparison, the spectra of pure AMP, pure MAPA, and the precipitates from AMP $50 \mathrm{wt} \%$ are also graphed.

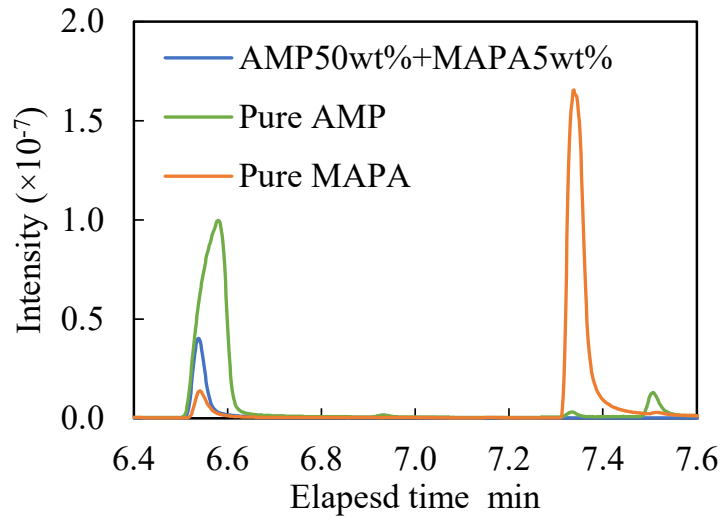

Figure 8. GC-MS spectra of precipitates from AMP $50 \mathrm{wt} \%+$ MAPA $5 \mathrm{wt} \%$ solution. Pure AMP and pure MAPA solution are graphed for comparison

\section{Mid-column Injection Process}

In our previous study of the phase separation process, separated $\mathrm{CO}_{2}$ semi-lean liquid was mixed with $\mathrm{CO}_{2}$ lean liquid and sent to the top of the absorber column. However, returning partially loaded solution (i.e., semi-lean liquid) to the top decreases the local absorption flux at the upper portion where is low $\mathrm{CO}_{2}$ partial pressure. By injecting semi-lean liquid into the middle of the absorber column, the local $\mathrm{CO}_{2}$ absorption driving force is maintained. This modification can improve the $\mathrm{CO}_{2}$ absorption rate.

Figure 9 shows the partial model of the absorber built on the process simulator Aspen Plus ${ }^{\circledR}$. The absorber column consisted of 10 stages. The change of $\mathrm{CO}_{2}$ recovery rate and precipitate quantity was simulated when the injection stage was changed stepwise from Stage 1 (top) to Stage 10 (bottom). Figure 10 shows the calculation results of AMP $50 \mathrm{wt} \%$ solution. The $\mathrm{CO}_{2}$ recovery rate and the precipitate quantity peaked when semi-lean liquid was injected at Stage 5, equivalent to the central portion of the absorber column. From this test results, the injection position of semi-lean liquid was set to the middle of the absorber column.

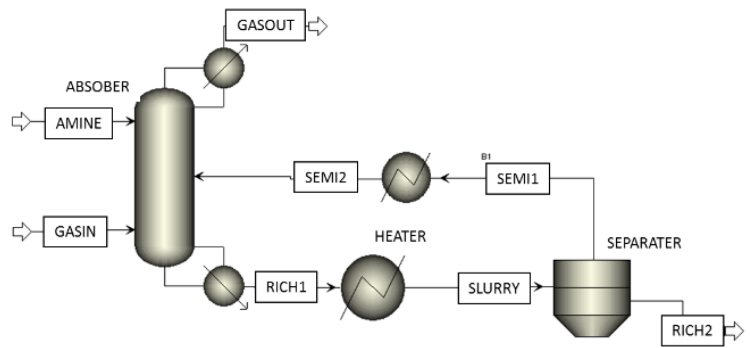

Figure 9. Partial model of absorber with variable injection location and precipitation of the $\mathrm{CO}_{2}$-rich phase

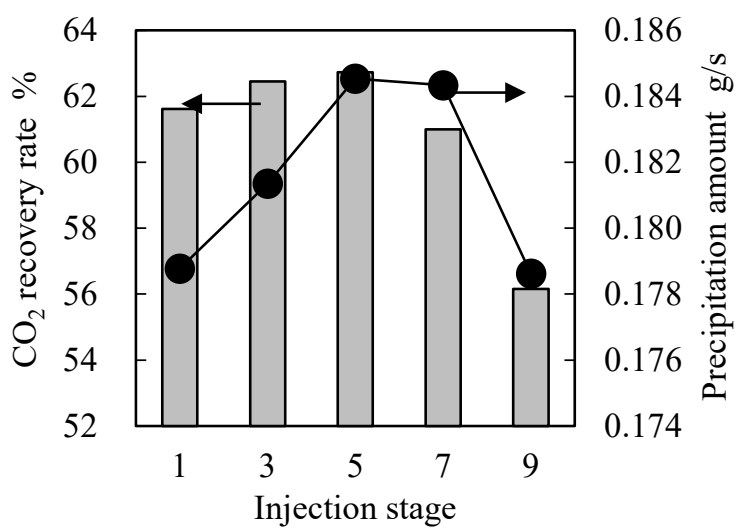

Figure 10. $\mathrm{CO}_{2}$ recovery rate and precipitation amount at each injection stage

\section{Evaluation by continuous $\mathrm{CO}_{2}$ Capture and Recovery Test}

\subsection{Experimental apparatus}

The new mixed amine and new injection location were evaluated by continuous $\mathrm{CO}_{2}$ capture and recovery tests using the apparatus shown in Figure 11. The main points in the process are highlighted by numbers (1-5) and letters $(\mathrm{S}, \mathrm{L})$ in circles; these points correspond to Figure 1. The new injection location is designated by (1)' in Figure 11

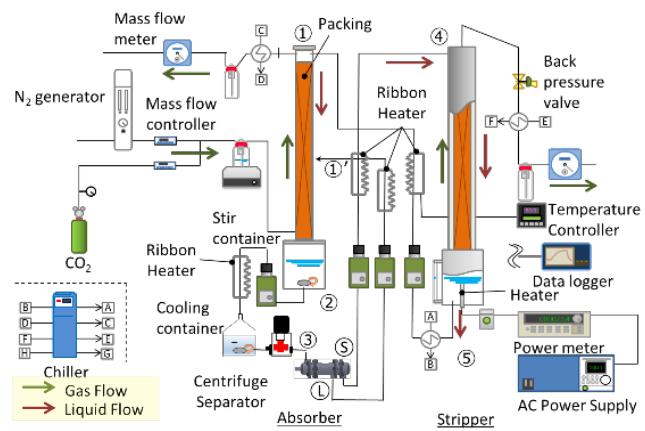

Figure 11. $10 \mathrm{~kg} /$ day-scale apparatus of $\mathrm{CO}_{2}$ capture and recovery 
The temperature in absorber must be maintained above the precipitation temperature to prevent passage blockage. To avoid precipitation prior to reaching the cooling container, the temperature of the lower of absorber and transfer lines to the cooling container were maintained at $65.0^{\circ} \mathrm{C}$. The cooling container was chilled at the precipitation temperature of around $53.0^{\circ} \mathrm{C}$ by $20.0^{\circ} \mathrm{C}$ brine with natural cooling without insulation.

The amine concentration in solution was reduced to 40 $\mathrm{wt} \%$ in the $\mathrm{CO}_{2}$ capture and recovery test because the amount of precipitate increases by lowering the amine concentration under the condition of a constant liquid gas ratio.

Table 1 shows this test conditions.

Table 1. Conditions of $\mathrm{CO}_{2}$ recovery tests using AMP $50 \mathrm{wt} \%+$ MAPA $5 \mathrm{wt} \%$

\begin{tabular}{|l|c|}
\hline Liquid gas ratio & 6 \\
\hline $\mathrm{CO}_{2}$ gas flow rate $[\mathrm{L} / \mathrm{min}]$ & 2.2 \\
\hline $\mathrm{N}_{2}$ gas flow rate $[\mathrm{L} / \mathrm{min}]$ & 12.7 \\
\hline $\mathrm{CO}_{2}$ partial pressure $[\mathrm{kPa}]$ & 15 \\
\hline Upper stripper temperature $\left[{ }^{\circ} \mathrm{C}\right]$ & 110 \\
\hline Lower stripper temperature $\left[{ }^{\circ} \mathrm{C}\right]$ & 120 \\
\hline Electric power input of heater $[\mathrm{W}]$ & 250 \\
\hline
\end{tabular}

\subsection{Operation stability}

Operation stability of $\mathrm{CO}_{2}$ capture and recovery test including continuous precipitation and centrifugal separation was checked by material balance of $\mathrm{CO}_{2}$. Material balance of $\mathrm{CO}_{2}$ can be evaluated by two way which are the $\mathrm{CO}_{2}$ captured in the absorber, and $\mathrm{CO}_{2}$ gas flow rate from the outlet of stripper, shown in Table 2 . The difference between these flow rates was about $1.0 \%$, and it was confirmed that the experiment was operated stably.

Table 2. $\mathrm{CO}_{2}$ flow rate and difference between lean and rich loading of solution.

\begin{tabular}{|l|c|}
\hline $\mathrm{CO}_{2}$ captured in the absorber $[\mathrm{g} / \mathrm{h}]$ & 217 \\
\hline $\begin{array}{l}\mathrm{CO}_{2} \text { gas flow rate from the outlet of stripper } \\
{[\mathrm{g} / \mathrm{h}]}\end{array}$ & 215 \\
\hline
\end{tabular}

\subsection{Regeneration heat and $\mathrm{CO}_{2}$ recovery rate}

Regeneration heat $Q_{i n}$ in this test was measured by electrical energy of the heater at the bottom of stripper compensating inherent heat loss of the stripper $Q_{\text {loss }}$. The $\mathrm{CO}_{2}$ dissociation heat $Q_{R}\left[\mathrm{GJ} /\right.$ ton- $\left.-\mathrm{CO}_{2}\right]$ was obtained by Eq. (2), where $Q_{V}$ is the vaporization heat, $Q_{H}$ is the sensible heat.

$$
Q_{R}=Q_{i n}-Q_{V}-Q_{H}-Q_{\text {loss }}
$$

$Q_{V}$ was obtained by Eq. (3), where the stripper vapor flow rate is given by $W_{V}[\mathrm{~kg} / \mathrm{h}]$, the latent heat is $H_{V}[\mathrm{MJ} / \mathrm{kg}]$, and the recovery $\mathrm{CO}_{2}$ flow rate is $W_{\mathrm{CO} 2}\left[\mathrm{~kg}-\mathrm{CO}_{2} / \mathrm{h}\right]$.

$$
Q_{V}=W_{V} H_{V} / W_{\mathrm{CO} 2}
$$

The sensible heat was obtained by Eq. (4), where the liquid flow rate is $W_{S}[\mathrm{~kg} / \mathrm{h}]$, specific heat is $C_{p}[\mathrm{MJ} / \mathrm{kg} / \mathrm{K}]$, the stripper inlet temperature is $T_{i n}[\mathrm{~K}]$, and the stripper outlet temperature is $T_{\text {out }}[\mathrm{K}]$.

$$
Q_{H}=W_{S} C_{p}\left(T_{\text {out }}-T_{\text {in }}\right) / W_{\mathrm{CO} 2}
$$

Figure 12 shows the breakdown of regeneration heat of AMP $40 \mathrm{wt} \%+$ MAPA $5 \mathrm{wt} \%$ in comparison with the previous results of AMP $50 \mathrm{wt} \%+\mathrm{PZ} 5 \mathrm{wt} \%$. The sensible heat of this test was $0.48 \mathrm{GJ} /$ ton- $\mathrm{CO}_{2}$, which corresponded to a decrease of $11-39 \%$ against the result of AMP 50 $\mathrm{wt} \%+\mathrm{PZ} 5 \mathrm{wt} \%$ under the same condition.

The $\mathrm{CO}_{2}$ recovery rate was obtained by the supplied $\mathrm{CO}_{2}$ gas flow rate and $\mathrm{CO}_{2}$ captured in the absorber. The supplied $\mathrm{CO}_{2}$ gas flow rate was $2.2 \mathrm{~L} / \mathrm{min}$, which corresponds to $240 \mathrm{~g} / \mathrm{h}$. Since $\mathrm{CO}_{2}$ captured in the absorber was $217 \mathrm{~g} / \mathrm{h}$, the $\mathrm{CO}_{2}$ recovery rate was $90 \%$.

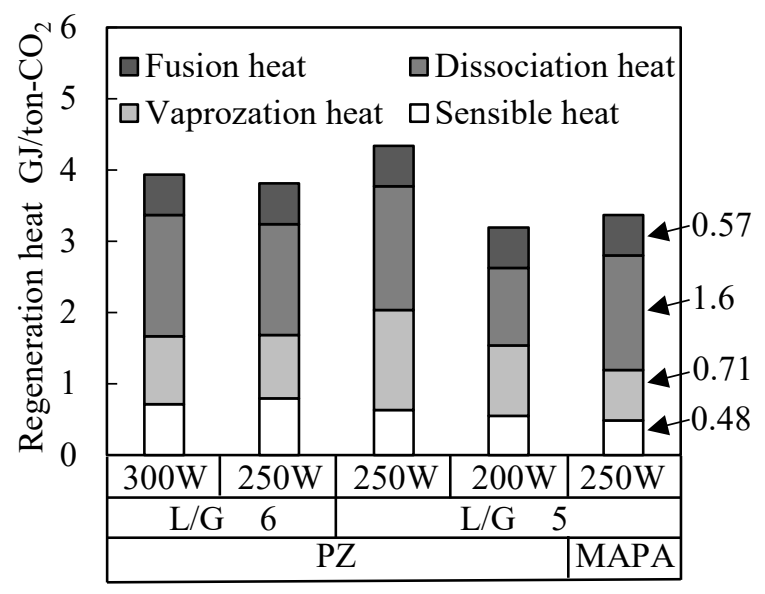

Figure 12. Regeneration heat of AMP $50 \mathrm{wt} \%+\mathrm{PZ} 5 \mathrm{wt} \%$ and AMP $40 \mathrm{wt} \%+$ MAPA $5 \mathrm{wt} \%$

\section{Conclusion}

This study aimed to improve the $\mathrm{CO}_{2}$ recovery rate via selecting new promoter and modification of absorber configuration and cooling condition in the phase separation process using high-concentration AMP. The reaction rate test showed higher $\mathrm{CO}_{2}$ absorption rate by adding MAPA than that by adding PZ, as such, AMP/MAPA solution was selected as a new solution. Simulation of the absorber column indicated that the highest $\mathrm{CO}_{2}$ recovery rate and precipitate quantity were obtained by sending semi-lean liquid to central portion of the absorber column. In $\mathrm{CO}_{2}$ capture and recovery tests with the new solution and the mid-column injection of semi-lean liquid, $\mathrm{CO}_{2}$ recovery rate reached to $90 \%$ and the sensible heat was reduced to $0.48 \mathrm{GJ} /$ ton $-\mathrm{CO}_{2}$.

\section{References}

Bernhardsen, I. and H. Knuutila; “A Review of Potential Amine Solvents for $\mathrm{CO}_{2}$ Absorption Process: Absorption 
Capacity, Cyclic Capacity and pKa," International Journal of Greenhouse Gas Control, 61, 27-48 (2017a)

Bernhardsen, I., I. Krokvik, K. Jens, and H. Knuutila; "Performance of MAPA Promoted Tertiary Amine Systems for $\mathrm{CO}_{2}$ Absorption: Influence of Alkyl Chain Length and Hydroxyl Group," Energy Procedia, 114, 1682-1688 (2017b)

Dash, S., A. Samanta, and S. Bandyopadhyay; "(Vapour + liquid) Equilibria (VLE) of CO2 in Aqueous Solutions of 2-amino-2-methyl-1-propanol: New Data and Modelling Using eNTRL-equation," The Journal of Chemical Thermodynamics, 43, 1278-1285 (2011)

Inoue, S., T. Itakura, T. Nakagaki, T. Furukawa, H. Sato, and Y. Yamanaka; "Experimental Study on $\mathrm{CO}_{2}$ Solubility in Aqueous Piperazine/alkanolamines Solutions at Stripper Conditions," Energy Procedia, 37, 1751-1759 (2013)

IPCC, 2018: Global Warming of $1.5^{\circ}$ C.An IPCC Special Report on the Impacts of Global Warming of $1.5^{\circ} \mathrm{C}$ above Pre-industrial Levels and Related Global Greenhouse Gas Emission Pathways, in the Context of Strengthening the Global Response to the Threat of Climate Change, Sustainable Development, and Efforts To Eradicate Poverty, V. Masson-Delmotte, P. Zhai, H.-O. Pörtner, D. Roberts, J. Skea, P. R. Shukla, A. Pirani, W. MoufoumaOkia, C. Péan, R. Pidcock, S. Connors, J. B. R. Matthews, Y. Chen, X. Zhou, M. I. Gomis, E. Lonnoy, T. Maycock, M. Tignor, and T. Waterfield eds (2018) in press

Kobari, M.; "New Interpreting Crystallization Process and Solvent-mediated Polymorphic Transformation," JGC Technical Journal, 6, No.4 (2014)

Nakagaki, T., S. Inoue, S. Sato, Y. Furukawa, and H. Sato; "Evaluation of Energy in Precipitating 2-Amino-2methyl-1-propanol Carbonate Solvent Process for $\mathrm{CO}_{2}$ Capture," Post-Combustion Capture Conference 2, 1a-2, Bergen, Norway (2013)

Nakagaki, T., S. Sato, H. Sato, and Y. Yamanaka; "Experimental Measurement of Regeneration Energy in $\mathrm{CO}_{2}$ Capture System Applying Phase Separation Process using high-concentration 2-Amino-2-methyl-1-propanol," Post-Combustion Capture Conference 3, 1B-2, Regina, Canada (2015)

Ogiyama, N., T. Hiro, H. Sato, J. Arakawa, and T. Nakagaki; "Stable Operation by Bench-scale $\mathrm{CO}_{2}$ Capture Apparatus Applying Phase Separation Process," The Society of Chemical Engineers, Japan $49^{\text {th }}$ Autumn Meeting., CA214, Nagoya, Japan (2017)

UNFCCC, V.; "Adoption of the Paris agreement." I: Proposal by the President (Draft Decision), United Nations Office, Geneva (Switzerland), (s 32). (2015)

Teranishi, H., H. Sato, J. Arakawa, Y. Yamanaka, and T. Nakagaki; "Modelling the Improvement of $\mathrm{CO}_{2}$ Capture Efficiency by Way of a Novel $\mathrm{CO}_{2}$-AMP Crystallization and Precipitation Process Using Aspen Plus," The Society of Chemical Engineers, Japan 48th Autumn Meeting., U121, Tokushima, Japan (2016)

Ying, J., S. Raets, and D. Eimer; "The Activator Mechanism of Piperazine in Aqueous Methyldiethanolamine Solutions." Energy Procedia, 114, 2078-2087 (2017)

Zhang, R., X. Luo, Q. Yang, H. Yu, G. Puxty, and Z. Liang; "Analysis for the Speciation in $\mathrm{CO}_{2}$ Loaded Aqueous MEDA and MAPA Solution Using ${ }^{13} \mathrm{C}$ NMR Technology," International Journal of Greenhouse Gas Control, 71, 1-8 (2018) 